\title{
Industrial Recommendation of Modeling of Inverter Based Generators for Power System Dynamic Studies with Focus on Photovoltaic
}

\author{
Koji Yamashita, Member, IEEE, Herwig Renner, Member, IEEE, Sergio Martínez Villanueva, \\ Gustav Lammert, Student Member, IEEE, Petros Aristidou, Member, IEEE, José Carvalho Martins, Member, IEEE, \\ Lingzhi Zhu, Luis David Pabón Ospina, Member, IEEE, and Thierry Van Cutsem, Fellow, IEEE
}

\begin{abstract}
Dynamic simulations have played an important role in assessing the power system dynamic studies. The appropriate numerical model is the key to obtain correct dynamic simulation results. In addition, the appropriate model including the selection of the individual model component (such as protections, controls and capabilities) is different depending on the type of phenomena to be observed or examined. However, the proper selection of the model is not an easy task especially for Inverter Based Generators (IBGs). Considerable industry experience concerning power system dynamic studies and the dynamics of the IBGs is required for the proper selection of the IBG model. The established CIGRE C4/C6.35/CIRED Joint Working Group (JWG) has gathered a wide variety of experts which fully cover the required industry experience. The JWG provides the guidance on the model selection for analyzing the phenomena such as frequency deviation, large voltage deviation, and long-term voltage deviation, individually. This helps to reduce the computational burden as well as it clarifies the required characteristics/functions that should be represented for the power system dynamic studies with the IBGs.
\end{abstract}

Index Terms-Capabilities, controls, dynamic models, generic models, inverter based generator, modeling, PhotoVoltaic (PV), protections, Root Mean Square (RMS) models, stability.

\section{INTRODUCTION}

$\mathbf{I}^{\mathrm{N}}$ $\mathrm{N}$ recent decades, inverter-based generators (IBGs) such as modern wind turbine generators (WTGs) and photovoltaic (PV) systems, have spread around the world in response

K. Yamashita is with the Dept. of Power System, System Engineering Laboratory, Central Research Institute of Electric Power Industry (CRIEPI), Kanagawa, Japan; e-mail: koji.yamashita.jp@ieee.org

H. Renner is with the Dept. of Institute of Electrical Power Systems, Graz University of Technology, Graz, Austria; e-mail: herwig.renner@tugraz.at

S. Martínez Villanueva is with the Dept. of Power System Reliability, Red Eléctrica de España, Alcobendas, Spain; e-mail: smartinez@ree.es

G. Lammert is with the Dept. of Energy Management and Power System Operation, University of Kassel, Kassel, Germany; e-mail: gustav.lammert@uni-kassel.de

P. Aristidou is with the School of Electronic \& Electrical Engineering, The University of Leeds, UK; e-mail: p.aristidou@ieee.org

L. Zhu is with the Renewable energy research center of China Electric Power Research Institute (CEPRI), Nanjing, China; e-mail: zhulingzhi@epri.sgcc.com.cn

J. Carvalho Martins is with the Dept. of Asset and Network Planning, EDP Distribuição, Lisbon, Portugal; e-mail: josecarvalho.martins@edp.pt

L. D. Pabón Ospina is with the Dept. of System Stability and Grid Integration, Fraunhofer Institute for Energy Economics and Energy System Technology (IEE), Kassel, Germany; e-mail: luis.david.pabon.ospina@iee.fraunhofer.de

T. Van Cutsem is with the Fund for Scientific Research (FNRS) at the Dept. of Electrical Engineering and Computer Science, University of Liège, Liège, Belgium; e-mail: t.vancutsem@ulg.ac.be to the commitment by numerous governments to increase renewable energy production to deal with global warming and other environmental concerns. In the past, the dynamics and resulting security margins of power systems were largely determined by the characteristics of (large) synchronous generators connected at the transmission system level, whereas nowadays, the impact of IBGs and their specific characteristics can no longer be ignored.

While the penetration level of IBGs was low, their impact on power system security and performance was negligible. In contrast, transmission system operators (TSOs) and distribution system operators (DSOs) today are facing operational situations where the penetration of IBGs is exceeding $50 \%$. A number of power systems are at times operating with over $60 \%$ of the instantaneous load demand being supplied from IBGs. The increasing penetration of IBGs will affect the resilience of networks to withstand a wide range of contingency events with different levels of probability if they are not integrated appropriately. This is in part due to the displacement of conventional large synchronous generators and their stabilizing controls. Many existing IBG technologies do not provide the same characteristics that synchronous generators have traditionally contributed to the power system, including inertia and significant fault current provisions. This has driven some grid codes to now require that new IBGs contribute to power system stability and operation by incorporating certain ancillary services such as voltage and frequency control.

Dynamic simulations have played an important role for many years in assessing the stability and security of power systems. Such studies are performed by power system planners and operators by means of numerical models and their associated software products. To do so, tailored dynamic models representing all critical elements in the power system are developed, with model complexity adjusted to account for the physical phenomena being investigated. Models for synchronous generators and their associated controls have been developed over many years and are well understood. In comparison, a limited number of available generic models can be used to represent the various types of IBGs, especially mini and micro installations that represent a growing percentage of embedded (distributed) generation. Industry research indicates that wind and PV generation is very likely to be modelled by utilities and system operators for power system dynamic studies with $76 \%$ and $67 \%$, respectively [1]. However, $35 \%$ 
of utilities and system operators still use negative load models to represent IBGs in power system dynamic studies [2]. According to the research results, the reasons for this approach are:

- Lack of well-defined model requirements for IBGs for specific power system phenomena.

- Limited access to well-validated, detailed IBG models.

- Lack of widely accepted generic IBG models and associated parameters.

- Lack of grid code requirements.

- Lack of information about the power system at the lower voltage levels associated with distribution and subtransmission networks.

- Lack of an accepted (agreed) methodology for the aggregation of distributed IBGs.

- Insufficient knowledge and experience in the practical operation of IBGs in the power system.

It is noted that the negative load models may be used when the penetration level of IBGs is very low and the impact of IBGs on the dynamic performance of the system is negligible.

Significant efforts have been made in the past by modeling experts to establish generic root mean square (RMS) type models through such organizations as the International Electrotechnical Commission (IEC) in Europe and the Western Electricity Coordinating Council (WECC) in North America. The activities were mainly focused on the development of generic models for WTGs [3]-[5]. However, these generic models are not yet widely applied, especially in Europe. The CIGRE and CIRED JWG Modeling and dynamic performance of inverter based generation in power system transmission and distribution studies [6] was established in 2014 gathering a wide range of experts who fully covered the required industry experience for developing a set of recommendations for modeling IBGs, with the focus on photovoltaic systems.

This paper has cataloged the components and functions that need to be included in the IBG model, depending on the power system phenomena being studied. In total, twenty-five functions are classified into three IBG categories, i.e., control, protection and capability. The paper provides a reasonable indication of the relevance of each function for different types of power system stability studies. The necessity of each function is examined for the following five power system phenomena that are of common interest to system operators:

- Frequency deviations

- Large voltage deviations (associated with transient network faults)

- Small and long-term voltage deviations (smaller magnitude but longer duration of changes in network voltage)

- Small signal analysis (oscillatory stability and damping studies)

- Unintentional islanding events

The rest of the paper is organized as follows. Section II introduces the functions of IBGs that need to be modeled for frequency and voltage deviations. The modeling requirements for IBGs investigating unintentional islanding are described in Section III. Conclusions are presented in Section IV.
TABLE I

TYPE OF PHENOMENA AND STUDIES TYPICALLY PERFORMED USING RMS-TYPE MODELS

\begin{tabular}{|l|l|}
\hline Type of phenomena & Type of studies \\
\hline \hline \multirow{3}{*}{ Frequency deviation } & - Frequency regulation \\
& - Frequency stability \\
& - Transient stability \\
\hline \multirow{3}{*}{ Large voltage deviation } & - Short-term voltage stability \\
& - Transient stability \\
& - Fault current contribution \\
& - Low/high voltage ride-through \\
\hline Small and long-term voltage deviation & - Long-term voltage stability \\
\hline Small-signal stability analysis & - Small-disturbance angle stability \\
\hline Unintentional islanding & - Unintentional islanding detection \\
\hline
\end{tabular}

II. NECESSARY FUNCTIONS OF IBGS FOR FREQUENCY

DEVIATION, VOLTAGE DEVIATIONS AND SMALL SIGNAL ANALYSIS

The paper also discusses how certain functions may be crucial for performing one type of study but can be reasonably neglected when performing another type of study. A selection of representative power system dynamic studies is provided in Table I to demonstrate how certain power system phenomena interact. For example, large voltage deviations are relevant when considering short-term voltage stability, transient stability and low voltage ride-through (LVRT) or high voltage ride-through (HVRT) studies and there might be an overlap between these issues depending on the characteristics of the power system being considered.

difference with a model used for transient stability analysis.

The necessity of each model component is discussed, with focus on the impact that omitting certain functions may have when performing specific types of studies. Secondary model components, that is, components that need not be modeled, are also identified in the paper. It is noted that as long as the dynamic behavior of the IBG is sufficiently accurate for the type of phenomenon being studied, applying appropriate simplifications that exclude secondary components can help to reduce the computational burden when performing simulations.

The characteristics of IBGs are used to extract the necessary functions of the IBG model components, which can be classified into three categories (see Table II):

- Control: denotes the internal control of the inverter which is performed at a local level.

- Protection: denotes the protection relay for the inverter. The grid protection relay for the inverter is also included. The control for protecting the internal devices is also categorized as a protection function.

- Capability: denotes the grid-friendly control or the control that has the ability to improve the grid stability.

Small signal analysis is not explicitly shown in Table II (see footnotes $e$ ) and the three functions are merged in Table II (see footnotes $g$ ).

The paper lists the relevant phenomena simulated in power system dynamic studies and provides the functions that should be considered for each type of phenomenon. The list of phenomena and the list mapping to the type of studies are presented in Table I. The types of studies shown in Table I are based on the classification in [7]. 
TABLE II

FUNCTIONS OF IBGS TO BE CONSIDERED FOR TYPE OF PHENOMENA

\begin{tabular}{|c|c|c|c|c|c|}
\hline Category & Functions & $\begin{array}{l}\text { Frequency } \\
\text { deviations }\end{array}$ & $\begin{array}{l}\text { Large voltage } \\
\text { deviations } \mathrm{e}^{\mathrm{s}}\end{array}$ & $\begin{array}{l}\text { Small and long-term } \\
\text { voltage deviations }\end{array}$ & $\begin{array}{l}\text { Unintentional } \\
\text { islanding }\end{array}$ \\
\hline Control & DC source control & No & $\mathrm{Yes}^{\mathrm{a}}$ & No & Yes \\
\hline Control & Current control & No & Yes $^{\mathrm{a}}$ & No & Yes \\
\hline Control & Phase Locked Loop (PLL) & No & Yes & No & Yes \\
\hline Control & Maximum Power Point Tracking (MPPT) & Yes $^{\mathrm{b}}$ & No & Yes & No \\
\hline Protection & $\begin{array}{l}\text { Reduction of maximum inverter current } \\
\text { when the DC voltage overcomes a certain limit }\end{array}$ & No & Yes $^{\mathrm{a}}$ & No & Yes \\
\hline Protection & Limitation of inverter current's variation rate after faults & No & Yes & No & Yes \\
\hline Protection & Current limit & Yes & Yes & Yes & Yes \\
\hline Protection & DC over voltage protection & No & Yes $^{\mathrm{a}}$ & No & Yes \\
\hline Protection & Over/under voltage protection & No & Yes & Yes & Yes \\
\hline Protection & Over/under frequency protection & Yes & Yes & No & Yes \\
\hline Protection & Protection for detecting faults ${ }^{\mathrm{g}}$ & No & $\mathrm{N} / \mathrm{A}^{\mathrm{d}}$ & No & Yes \\
\hline Protection & $\begin{array}{l}\text { Rate Of Change Of Frequency (ROCOF) tripping: } \\
\text { monitoring the frequency variation rate and disconnecting } \\
\text { the inverter when it reaches a certain limit }\end{array}$ & Yes & Yes & $\mathrm{No}^{\mathrm{f}}$ & Yes \\
\hline Protection & Vector jump & Yes & Yes & No & Yes \\
\hline Protection & Transfer trip & No & No & No & Yes \\
\hline Protection & Anti-islanding active detection method & Yes & Yes & Yes & Yes \\
\hline Capability & Frequency control by means of active power & Yes & No & No & Yes \\
\hline Capability & Voltage control by means of reactive power & Yes & Yes & Yes & Yes \\
\hline Capability & Voltage control by means of active power & No & Yes & Yes & No \\
\hline Capability & Synthetic inertia & Yes & Yes & No & Yes \\
\hline Capability & Rate Of Change Of Frequency (ROCOF) immunity & Yes & No & No & Yes \\
\hline Capability & $\begin{array}{l}\text { Low Voltage Ride-Through (LVRT) and } \\
\text { High Voltage Ride-Through (HVRT) }\end{array}$ & No & Yes & No & Yes \\
\hline Capability & $\begin{array}{l}\text { Reactive power control in response to } \\
\text { fast and large voltage variations }\end{array}$ & No & Yes & No & Yes \\
\hline Capability & Power Oscillation Damping (POD) & Yes $^{\mathrm{c}}$ & Yes $^{\mathrm{a}}$ & No & No \\
\hline
\end{tabular}

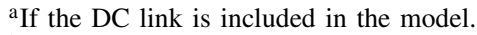

${ }^{\mathrm{b}}$ For small isolated systems.

${ }^{\mathrm{c}}$ If the damping controller is designed to damp the common mode of frequency evolution.

${ }^{\mathrm{d}}$ Not applicable. External protection relay model may be used such as zero sequence over voltage protection model for single line-to ground faults

${ }^{\text {e}}$ For small signal analysis, protections and limiters are excluded from this column.

${ }^{\mathrm{f}}$ For long-term voltage stability analysis considering emergency control actions, the ROCOF tripping becomes important especially when the immediate voltage change caused by such emergency control actions is no longer small.

${ }^{\mathrm{g}}$ Balanced fault, line-to-line fault, single-line-to-ground fault protection models need to be implemented in response to the intended type of fault.

\section{A. Functions in the category: Control}

1) DC source control: The DC source control is necessary for large voltage deviations at the DC link. If a system fault occurs and the voltage level is extremely low, the valve device in the inverter could be blocked, which is known as valve device blocking [8] or interruption of current injection [9]. In such a case, the DC voltage will rise because the DC source continues to provide DC current, while the AC current is controlled to zero. This could cause an overvoltage in the DC link and would trigger the DC over voltage protection. Without this DC overvoltage protection model, the simulated response of the active and reactive power can be different from the actual measured response.

2) Phase locked loop (PLL): The PLL control is necessary for large voltage deviations at the DC link. When large voltage deviations occur, the bus voltage angle could quickly and significantly change. Such an immediate jump of the voltage angle could delay tracking the voltage angle change at the point of interconnection. Such a time delay coming from the response speed of the PLL could result in undesirable transient positive or negative active or reactive power variations. Meanwhile, state-of-the-art IBGs may have very short time delays. The change in active and reactive power of the IBGs following system faults can affect the critical area exchange or the maximum power transfer with respect to transient stability or short-term voltage stability. Furthermore, the detailed PLL control block is usually modeled using the EMT model, and the simplified control block that approximates the PLL behaviour is normally modeled using the RMS model [10] because the assumption that the IBG model can be represented only by a current source can be justified by the fact that converter control is much faster than the dynamics/transients of interest.

3) Current control: The current control is necessary for large voltage deviations at the DC link. The converter model is simplified to a current controller model that generates the desired currents. The electrical controller of this type of model only extracts the essential component of a more detailed electrical controller, i.e. a decoupled active and reactive power controller via current control. The controller has a doubleloop structure. The outer loop uses the active power and reactive power references to generate the respective d-axis and q-axis current references for the inner loop. The inner loop regulates its current output to the respective current reference. In both control loops, PI controllers are usually used. It is often the case that the inner current control loop is also omitted in the RMS model mainly because the current control 
is most likely to be completed in the time step of the RMS simulation. Such a model is less complex than the detailed generator/converter and electrical control model; however, it focuses on the fundamental characteristics of the converter outputs and has been widely applied in many engineering projects for bulk power system dynamic studies.

4) Maximum power point tracking (MPPT): The MPPT is necessary for frequency deviations and long-term voltage deviations. The simulation time for frequency control and stability studies can be split into three different time scales: i) less than 30 seconds, ii) longer than 5 minutes, and iii) longer than 10 minutes. The first one is to examine the frequency nadir and the settled frequency in the case of a generator tripping. The second one is to examine the peak-to-peak value of the frequency fluctuation in a small isolated system. The third one is to examine long-term voltage deviations. When ii) and iii) need to be examined, the change in the MPPT signal needs to be considered for this type of phenomenon. This is because the solar radiation cannot be assumed to be constant for the aforementioned time scale. However, the use of the MPPT model element is not always necessary because the control response speed is fast enough for the long-term dynamics. Therefore, the change in MPPT signal may be modeled as a change in the active power reference itself.

\section{B. Functions in the category: Protection}

1) Reduction of maximum inverter current when the DC voltage overcomes a certain limit; and DC over voltage protection: Refer to the descriptions for DC source control in Section II-A1.

2) Limitation of inverter current's variation rate after a fault: This protection is necessary for large voltage deviations. The rate of current variation needs to be designed to be below the maximum permissible value of the inverter switching devices. It is often set by selecting appropriate values for the inductance in the main circuitry and therefore it is by design a constant. In some systems, an adjustable current variation rate exists, which can be implemented within the current control loop to protect the switching devices from excessive current stresses. When a short-circuit occurs in the power system, excessive current will flow through the switching devices, resulting in extra stress on the devices. If the temperature at the junctions of the switching devices rises higher than the fixed internal threshold with hysteresis, the current variation rate will be reduced for a certain length of time to protect the switching devices.

Because the operation of this type of protection will lead to the massive disconnection of IBGs, this could affect the critical area exchange or the maximum power transfer with respect to transient stability or short-term voltage stability.

3) Current limit: This protection is necessary for all phenomena. When the frequency drops, it is desirable for any generator to increase its active power output to mitigate the frequency nadir. On the other hand, the active and reactive power output of the IBG is limited by the maximum current. Therefore, the increasing amount of active power output of the IBG during the frequency drop might be limited not only because there is no available headroom, but also because the allowable current from the IBG is limited. This means that the different logic for the limitation of the current could lead to a different increasing amount of the active power output. It will also result in a different dynamic frequency response. Therefore, the current limit needs to be modeled for the frequency deviation.

In the case of small isolated systems, the number of synchronous operating units could dramatically decrease due to the integration of IBGs. For example, consider two synchronous operating units in a small isolated system. When one of the two units is disconnected from the grid, not only does a significant frequency drop occur, but a significant voltage drop also occurs. If the IBGs generate less than $100 \%$ (of the rated current), say $70 \%$, their active power output may be assumed as constant. Conversely, if the IBGs are operated at almost $100 \%$, their active power output cannot increase and will decrease when the terminal voltage of the operating unit drops, which will cause a larger frequency drop.

When the voltage drop is significant, the active or reactive power output of the IBG can increase quickly. This means that the current of the IBG is more likely to hit its limit and the active or reactive power of the IBG will be restricted so as to control the current of the IBG within a permissible range. It should be noted that the dynamic behavior of the active and reactive power of the IBG could be different because the control scheme can vary depending on the IBG and the grid code. The possibility of hitting the current limit also depends on the initial current of the IBG, i.e., the pre-fault current. If the initial current of the IBG is high, the recovery of the active power or the increase in reactive power is more likely to be limited, which would result in a different critical area exchange or a different maximum power transfer and therefore could affect transient or short-term voltage stability.

When the power system is about to experience long-term voltage instability, any significantly low voltage at a system bus must be monitored. Extremely low voltages could lead to a current limitation of the IBG due to a high current injection.

4) Over/under voltage protection: This protection is necessary for voltage deviations. If a system fault occurs and if the fault duration is long, the under voltage protection for both loads and IBGs is likely to trip. After the fault is cleared voltage recovery may be of a long duration (e.g. due to the massive induction motor loads) and the under voltage protection is likely to trip. The dynamic reactive power (current) support of the IBGs could cause a significant voltage rise after the fault is cleared [11]. The self-disconnection of loads [12] with the active power recovery of the IBGs could also cause a significant voltage rise after the fault is cleared [13]. Such significant voltage rise is most likely to trip the overvoltage protection, which would lead to different critical area exchange or different maximum power transfer and hence could impact transient or short-term voltage stability.

When the power system is about to experience long-term voltage instability, any significantly low voltage at a system bus must be observed. Extremely low voltages could trip the undervoltage protection. 
5) Over/under frequency protection: This protection is necessary for frequency and large voltage deviations. The large integration of IBGs could cause a larger undesired frequency nadir especially in the case of a small electrical island or the loss of a large generation unit. This could lead to tripping of the over/underfrequency protection. In addition to this relay operation, automatic reconnection should also be considered.

If a large power plant is connected to the main grid via a transmission line and a short-circuit occurs on that transmission line, not only could a large voltage deviation occur, but a significant frequency deviation could also occur. When massive amounts of residential and utility-scale IBGs, with improperly designed LVRT/HVRT capability and/or PLL, disconnect following a system fault, significant frequency drops can be observed [9]. If loads are disconnected due to the underfrequency load shedding scheme, an overfrequency could also occur. Therefore, the over- or underfrequency protection might operate in the case of large voltage deviations with a high penetration of IBGs.

6) Protection for detecting balanced faults; protection for detecting unbalanced faults; and protection for detecting single-line-to-ground faults: This protection may be used for large voltage deviations. The decrease of short-circuit power in the system, due to the smaller fault current contributions of IBGs compared to synchronous generators, could be a future issue and such protection needs to be modeled to examine the successful relay operation.

7) Rate Of Change Of Frequency (ROCOF) tripping: monitoring the frequency variation rate and disconnecting the inverter when it reaches a certain limit: ROCOF tripping should be modeled for frequency deviations and large voltage deviations. The large integration of IBGs could cause a larger undesired frequency nadir especially following a significant increase/decrease in load or generation.

The frequency is normally measured using the bus voltage. When a system fault occurs, the bus voltage angle could immediately shift to a different voltage bus angle [9]. Such a "jump" of the voltage angle could also cause a significantly large ROCOF value. To avoid the undesirable ROCOF relay operation, a filter may be used. However, this can result in slower operation of the ROCOF relay in the case of a real frequency drop. The ROCOF tripping is closely related to frequency deviations. However, frequency instability could occur together with transient instability through the high penetration of IBGs. Therefore, the ROCOF relay might operate due to the large voltage deviation with a high penetration of IBGs.

8) Vector jump: This protection is necessary for frequency and large voltage deviations. The vector jump method is a typical passive anti-islanding protection scheme. The fundamental principle is to detect the islanded condition via a change in the voltage phase. Generally, one of the assumed triggered events for frequency deviations is a generator tripping. Not only the generator tripping but also the disconnection of IBGs and the interruption of their current injection can be the triggering event. As shown in Fig. 1a, when the active power flow changes dramatically without a significant change in the system voltage, the angle between two buses can exhibit the step change $\Delta \delta_{2}$ when the generator trips:

$$
\begin{aligned}
& \left\{\begin{array}{l}
P=\frac{V_{1} V_{2}}{X} \sin \left(\delta_{2}-\delta_{1}\right) \\
P-\Delta P=\frac{V_{1} V_{2}}{X} \sin \left(\delta_{2}-\Delta \delta_{2}-\delta_{1}\right)
\end{array}\right. \\
& \therefore \Delta P \simeq \frac{V_{1} V_{2}}{X} \Delta \delta_{2}
\end{aligned}
$$

Such an immediate angle change can cause undesired vector jump operation and the disconnection of IBGs, which could lead to a further frequency drop.

It is highly possible that the voltage phase angle will jump to another voltage phase angle when the network is split into multiple grids. On the other hand, the voltage phase angle could jump due to a change in the network configuration although the network is not separated. A typical change in the system configuration is during a system fault that has a large voltage deviation, i.e., when the system fault occurs and when the system fault is cleared. As mentioned earlier, the voltage phase angle jump can lead to a significant change in frequency seen by the IBG, which could lead to disconnection of the IBG [9]. Hence, such disconnection of IBGs may be represented by the vector jump protection as well as the over/underfrequency protection.

9) Anti-islanding active detection method: The antiislanding active detection method should be modeled for all phenomena. Anti-islanding active detection methods intentionally inject negative/positive reactive power into the network, which assists the growing frequency change/deviation when the designated system is isolated from the main grid. A small proportion of anti-islanding active detection methods utilize the GPS signal or system frequency for determining the control signal for the positive/negative reactive power injection. IBGs that are equipped with such anti-islanding active detection methods could inject positive/negative reactive power in synchronization with each other. Due to the voltage sensitivity of loads, an increase in voltage caused by an increase in reactive power via the anti-islanding protection method, will lead to an increase in the active power consumption of loads and a further system frequency drop.

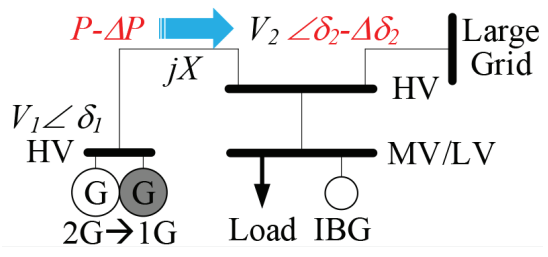

(a) Voltage phase jump by means of active power

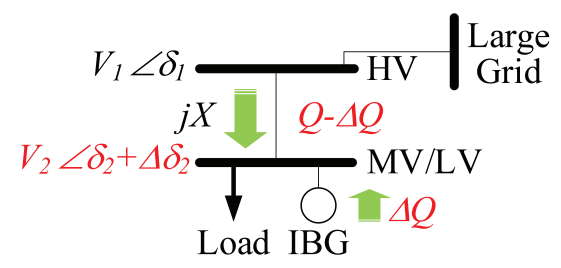

(b) Voltage phase jump by means of reactive power

Fig. 1. Voltage phase jump mechanism. 
Moreover, a frequency change that is derived from the bus voltage can also occur when the voltage magnitude changes due to the reactive power injection, no matter how sensitive the load is to voltage. As shown in Fig. 1b, when the small reactive power injection from the IBG does not have the ability to change the connected bus voltage, a voltage phase angle change occurs:

$$
\begin{aligned}
& \left\{\begin{array}{l}
Q=\frac{V_{1} V_{2}}{X} \cos \left(\delta_{2}-\delta_{1}\right)-\frac{V_{2}^{2}}{X} \\
Q-\Delta Q=\frac{V_{1} V_{2}}{X} \cos \left(\delta_{2}+\Delta \delta_{2}-\delta_{1}\right)-\frac{V_{2}^{2}}{X}
\end{array}\right. \\
& \therefore \Delta Q \simeq \frac{V_{1} V_{2}}{X}\left(\delta_{2}-\delta_{1}\right) \Delta \delta_{2}
\end{aligned}
$$

Therefore, if a positive reactive current is injected in the case of a frequency drop, a further system frequency drop could occur. On the other hand, if the penetration of IBGs is very high and if the cumulative reactive current injection from the distributed IBGs has ability to change the voltage magnitude, a negative reactive current injection during the voltage dip could lead to a further voltage drop (in short-term and long-term).

It should be noted that the anti-islanding active detection method is not widely used around the world and such a model does not need to be modeled for an IBG that is not equipped with the anti-islanding active detection method.

\section{Functions in the category: Capability}

1) Frequency control by means of active power: This capability is necessary only for frequency deviations. The increased utilization of tie-lines is encouraged in terms of increased integration of renewable energies. The increased tie-line power flow could lead to more significant frequency increases/decreases after a power system is split into two subsystems due to tie-line tripping. A frequency rise in the system could lead to the disconnection of a large amount of IBGs due to the overfrequency protection. On the other hand, the tripping of this large amount of generation can lead to a frequency drop and therefore a disconnection of load due to the underfrequency load shedding scheme. To overcome this problem, IBGs can control the frequency by means of active power, i.e., reduce their active power feed-in during overfrequency, or increase their active power feed-in during underfrequency. Hence, this function can play an important role when the power system experiences a significant frequency change. In this context, the following topics are addressed:

- Modeling the primary power source control (if existing) including maximum range and gradients (for frequency rise/drop)

- Modeling the optional storage devices including charging management (for frequency rise/drop)

- Modeling the power reserve in the case, where the IBG is operated at a point below the maximum power point in order to allow primary frequency control (for frequency drop only).

2) Voltage control by means of reactive power: This capability is necessary for all phenomena. Furthermore, it is generally required for the steady-state condition. Therefore, the dynamic reactive power (current) support during fault conditions is not included in this capability. Although, the dynamic behavior is not directly related to this capability, the pre-fault active and reactive power feed-in of the IBG can affect the fault-on and post-fault dynamic behavior of the IBG output. This could indirectly change the dynamic frequency response of the system. Furthermore, this behavior could also result in a different critical area exchange or a different maximum power transfer, which would affect the rotor angle and short-term voltage stability.

In general, this voltage control is intentionally set as slow as possible in order to coordinate with other voltage controllers, such as tap changers and reactive power compensators. Therefore, the operation of the voltage control by means of reactive power of IBGs can play an important role when the power system experiences long-term voltage instability.

3) Voltage control by means of active power: This capability is necessary for voltage deviations. Voltage control by means of active power is commonly used for residential PV systems. The IBG reduces its active power when the voltage exceeds the threshold value, e.g., 1.09 p.u. If a significant voltage dip occurs in the grid, induction motors might stall and loads could be disconnected from the power system. A large amount of self-disconnected loads can cause a voltage rise. The control speed of this type of voltage control is slow and the reduction of active power output from PV systems is in the order of tens of seconds to a minute. Therefore, transient stability could be affected by voltage control by means of active power if the simulation time considers the aforementioned study period.

Without large voltage deviations, voltage control by means of active power can be considered when the load decreases and/or the PV system output increases in the steady state. Therefore, voltage control by means of active power can play an important role when the power system experiences longterm voltage instability.

4) Synthetic inertia: This capability is necessary for frequency and large voltage deviations. As mentioned earlier, the displacement of synchronous generators by IBGs could cause a reduction of the system inertia. According to the power swing equation, the lower inertia will increase the change in rotor angle speed for the same mismatch between mechanical and electrical power. The increase in the ROCOF could endanger the frequency stability. IBGs will not exhibit inertia-like behavior per se [14], [15]. While some of the prime movers could deliver inertia, it is not certain that the IBG can provide it, because it would require a modification of the control [16]. However, assuming an appropriate control scheme of the inverter and (limited) energy storage capability, synthetic inertia can be provided to the grid. The potential solutions that can be used are:

- Installation of energy storage devices, e.g., large flywheel generators or flywheel coupled synchronous compensators.

- Adoption of alternative approaches to detect electrical islands and ensure that the main equipment is robust against ROCOF. 
- Operation of PV systems below the maximum power point.

- Modification of the control of IBGs to provide synthetic inertia, which can be done by a change in active power output during frequency deviations. This is the approach that has been followed by Hydro Quebec [17]. This technology is recognized as fast frequency response (FFR) and closed-loop inertia-based FFR has been commercially available for many years [18]. However, its practical use is still being discussed and requires careful examination and implementation [16].

Rotor angle stability consists of two elements: i) synchronizing torque in phase with rotor angle deviations, and ii) damping torque in phase with speed deviations [7]. The damping torque can be expressed as:

$$
\begin{aligned}
& D=\frac{2 M}{T_{d}} \\
& \because s=\frac{D}{2 M} \pm j \sqrt{\frac{\omega_{0} K}{M}-\frac{D^{2}}{4 M^{2}}} \\
& \because \Delta T_{m}=\frac{M}{\omega_{0}} s^{2} \Delta \delta+\frac{D}{\omega_{0}} s \Delta \delta+K \Delta \delta
\end{aligned}
$$

where:

$M$ denotes system inertia,

$D$ denotes damping torque,

$K$ denotes synchronizing torque,

$S$ denotes Laplace operator,

$T_{d}$ denotes decay time constant,

$T_{m}$ denotes mechanical torque.

The damping torque includes the system inertia $M$, which means the system inertia can affect the damping torque and therefore the rotor angle stability. Because the power swing oscillations caused by large voltage deviations can change with and without synthetic inertia, it could also influence the critical area exchange or the maximum power transfer with respect to the rotor angle and short-term voltage stability.

5) Rate Of Change Of Frequency (ROCOF) immunity: This capability is necessary for all phenomena. ROCOF immunity is equivalent to frequency ride-through, i.e., low and highfrequency ride-through. If the ROCOF setting for the ROCOF immunity is the same as the relay setting for the ROCOF protection (see Section II-B7), the ROCOF immunity model is not necessary. On the other hand, it is more likely that the relay setting for ROCOF protections could change depending on the location. In such a case, the ROCOF immunity needs to be modeled independently.

6) Low Voltage Ride-Through (LVRT) and High Voltage Ride-Through (HVRT): This function is necessary for large voltage deviations. The LVRT characteristic clarifies a zone where IBGs have to stay connected to the grid during grid disturbances depending on the duration and depth of the voltage sag. IBGs may trip if the voltage is outside the zone. The most conservative way from the viewpoint of stability is to trip the IBGs when the voltage is outside the zone. Thus, the LVRT characteristic is most likely to be represented as an under voltage protection with different settings for time delays and voltage thresholds. These protection settings ensure LVRT and HVRT capability. Although, different manufacturers have different control strategies for active and reactive power during the fault-on period, there are common principles. In general, when the voltage drops, the IBGs will reduce their active power output. In some countries, the active power output is not allowed to be zero during the fault unless the residual voltage is below the threshold value, e.g., 0.2 p.u. Hence, the IBG is allowed to temporarily stop the current injection when the residual voltage is extremely low. In other countries, the active power output is allowed to be zero, while the reactive power output is required to increase. When modeling the FRT characteristics, it is important to set up-to-date undervoltage limits according to the information provided by the manufacturer or the grid code requirements.

Because the dynamic behavior of the IBG output following faults can significantly change with and without the LVRT/HVRT characteristics, it could also affect the critical area exchange or the maximum power transfer and therefore the transient and short-term voltage stability.

7) Reactive power control in response to fast and large voltage variations: This function is necessary for large voltage deviations. It is typically known as dynamic reactive power (current) support or dynamic voltage support. As mentioned in Section II-C6, this capability supports the voltage during and following faults by injecting reactive current and reducing active current. Such dynamic voltage support can change the critical area exchange or the maximum power transfer and therefore influence transient and short-term voltage stability. The current limit function is closely related to this function during large voltage variations because the active and/or reactive currents are more likely to hit their limit during large voltage deviations. In such a case, the inverter control modes such as active power priority and reactive power priority play an important role in how the active and/or reactive current hits the current limit, through which the different dynamic behavior of the active and reactive power of IBGs can affect the power system dynamic stability.

8) Power oscillation damping (POD): This function is necessary for frequency and large voltage deviations. Although it is not currently implemented in IBGs except HVDC, this capability can help mitigate undamped power swing oscillations or act to shorten the decay time of damped power swing oscillations. Such mitigation of power swing oscillations following faults can change the critical area exchange or the maximum power transfer and therefore influence transient and short-term voltage stability.

On the other hand, if the loss of generation causes poorly damped power swing oscillations, POD plays an important role to ensure transient stability. Therefore, in the aforementioned case, and if POD is assumed to be implemented in IBGs, IBGs with POD should be modeled; otherwise, there is no need to model the POD.

For small disturbance angle stability analysis, protections and limiters are typically ignored when linearizing the dynamic model around the operating point. Therefore, the necessary functions for the small signal stability analysis are those for the large voltage deviation excluding protection related functions. For example, POD becomes necessary for small 
signal analysis when oscillation could be excited in a poorly damped system.

\section{NECESSARY FUNCTIONS OF IBGS FOR UNINTENTIONAL ISLANDING}

Because most of the functions are necessary for studies related to unintentional islanding, only the unnecessary functions are listed.

1) Maximum power point tracking (MPPT): Anti-islanding is most likely to be detected less than 2 or 3 seconds after the formation of an island. The MPPT reference varies depending on the change in solar radiation. In general, the change in the radiation is very slow and therefore MPPT is not assumed for examining anti-islanding detection.

2) Voltage control by means of active power: Antiislanding is most likely to be detected in less than 2 or 3 seconds after forming islanding. Voltage control by means of active power is generally triggered a few seconds after the voltage violation, say over 1.09 p.u. Therefore, before such control action, the islanding detection needs to be completed. In general, voltage control by means of active power is not assumed for examining unintentional islanding.

3) Power oscillation damping (POD): The POD is more likely to be associated with HVDC, mainly to improve the damping of power swing oscillations. Power swing oscillations generally occur between two groups of generators. Once the electrical island is established, at least one group of generators disappears in the islanded system and power swing oscillations no longer exist in the islanded network.

\section{CONCLUSIONS}

All numerical models have their limitations. The identification of necessary functions for a specific dynamic behavior is the key to achieving realistic results. Modeling experts around the world, contributed to the CIGRE C4/C6.35/CIRED JWG and defined the necessary functions of IBGs, which should be considered in the models for studying specific power system phenomena. This paper extracted several major phenomena for power system dynamic studies with IBGs: i) frequency deviations, ii) large voltage deviations, and iii) small and long-term voltage deviations. To obtain appropriate simulation results for each type of power system dynamic study, the necessary functions of IBGs that should be implemented in the model, are defined and listed. Furthermore, the relationship between different power system phenomena and various types of power system dynamic studies is illustrated.

The required functions are classified into three categories: i) control, ii) protection, and iii) capability. Detailed clarifications of the necessity of each function is also provided. Generally, it can be concluded that the protection model needs to be more involved in many power system dynamic studies mainly because the operation of the protection could directly cause the disconnection of IBGs.

This guideline proposes dynamic models with associated functions that should be used for specific power system phenomena. The outlined recommendations in selecting the adequate model for IBGs are beneficial for both academia and industry. It should be noted that in some cases, secondary functions may also need to be modeled, even if not recommended in this paper.

The guideline proposed in this paper are expected to be reinforced in the future with additional experience in the operation of power systems including IBGs, new technical standards, revised grid code requirements, further research contributions, etc. In this context, the aggregation of distributed IBGs and its representation in power system dynamic studies, is crucial.

\section{ACKNOWLEDGMENT}

The authors would like to thank all 67 members who have been involved in the CIGRE C4/C6.35/CIRED JWG.

\section{REFERENCES}

[1] G. Lammert, K. Yamashita, L. D. Pabón Ospina, H. Renner S. Martínez Villanueva, P. Pourbeik, F.-E. Ciausiu, and M. Braun, "Modelling and Dynamic Performance of Inverter Based Generation in Power System Studies: An International Questionnaire Survey," CIRED - Open Access Proceedings Journal, vol. 1, pp. 1-5, 2017.

[2] G. Lammert, K. Yamashita, L. D. Pabón Ospina, H. Renner, S. Martínez Villanueva, P. Pourbeik, F.-E. Ciausiu and M. Braun, "International Industry Practice on Modelling and Dynamic Performance of Inverter Based Generation in Power System Studies," CIGRE Science \& Engineering, vol. 8, pp. 25-37, June 2017.

[3] Western Electricity Coordinating Council (WECC) Renewable Energy Modeling Task Force. (2012, September) WECC Solar PV Dynamic Model Specification. [Online]. Available: https://www.wecc.biz/Reliability/ WECCSolarPVDynamicModelSpecification-September2012.pdf.

[4] CIGRE Working Group C4.601, "Modeling and Dynamic Behavior of Wind Generation as it Relates to Power System Control and Dynamic Performance," Technical Brochure 328, pp. 1-216, August 2007.

[5] International Electrotechnical Commission (IEC), "Wind turbines Electrical simulation models for wind power generation," IEC 6140027-1, pp. 1-94, Feb. 2015.

[6] Homepage CIGRE SC C4. (2014) JWG C4/C6.35/CIRED Modelling and dynamic performance of inverter based generation in power system transmission and distribution studies. [Online]. Available: http://c4.cigre.org/content/view/full/40401

[7] P. Kundur, J. Paserba, V. Ajjarapu, G. Andersson, A. Bose, C. Canizares, N. Hatziargyriou, D. Hill, A. Stankovic, C. Taylor, T. Van Cutsem, and V. Vittal, "Definition and classification of power system stability ieee/cigre joint task force on stability terms and definitions," IEEE Trans. Power Syst., vol. 19, no. 3, pp. 1387-1401, Aug 2004.

[8] International Electrotechnical Commission (IEC), "Amendment 1 - International electrotechnical vocabulary - Part 551-20: Power electronics - Harmonic analysis,” IEC 60050-551-20:2001, pp. 1-29, July 2001.

[9] North American Electric Reliability Corporation (NERC). (2017, June) 1200 MW Fault Induced Solar Photovoltaic Resource Interruption Disturbance Report - Southern California 8/16/2016 Event. [Online]. Available: http://www.nerc.com/pa/rrm/ea/1200_ MW_Fault_Induced_Solar_Photovoltaic_Resource_/1200_MW_Fault_ Induced_Solar_Photovoltaic_Resource_Interruption_Final.pdf

[10] K. Clark, R. A. Walling, and N. W. Miller, "Solar photovoltaic (PV) plant models in PSLF," in 2011 IEEE Power and Energy Society General Meeting, July 2011, pp. 1-7.

[11] I. Green, "Caiso experience with impact of high penetration of renewable resources on short-term voltage stability," in 2015 IEEE Power Energy Society General Meeting, July 2015, pp. 1-18.

[12] CIGRE Working Group C4.605, "Modelling and Aggregation of Loads in Flexible Power Networks," Technical Brochure 566, pp. 1-191, February 2014.

[13] K. Yamashita, Y. Kitauchi, and H. Kobayashi, "Influence of voltage sags on the power system with high penetration of photovoltaic power generation," in 2012 IEEE Power and Energy Society General Meeting, July 2012, pp. 1-7.

[14] D. Consulting. (2016, Oct.) International review of frequency control adaptation. [Online]. Available: https://www.aemo.com. au/-/media/Files/Electricity/NEM/Security_and_Reliability/Reports/ FPSS---International-Review-of-Frequency-Control.pdf. 
[15] G. Lammert, K. Yamashita, H. Renner, S. Martínez Villanueva, J. Carvalho Martins, P. Aristidou, T. Van Cutsem, L. D. Pabón Ospina, M. Braun, and J. C. Boemer, "Activities of the Joint Working Group CIGRE C4/C6.35/CIRED: Modelling and Dynamic Performance of Inverter Based Generation in Power System Transmission and Distribution Studies," in 1st International Conference on Large-Scale Grid Integration of Renewable Energy in India, New Delhi, September 2017, pp. 1-8.

[16] N. Miller. (2017, March) Final Report: Technology Capabilities for Fast Frequency Response. [Online]. Available: https://www. aemo.com.au/-/media/Files/Electricity/NEM/Security_and_Reliability/ Reports/2017-03-10-GE-FFR-Advisory-Report-Final---2017-3-9.pdf.

[17] M. Asmine and C.-E. Langlois, "Field measurements for the assessment of inertial response for wind power plants based on hydro-quebec transenergie requirements," IET Transactions on Renewable Power Generation, vol. 10, no. 1, pp. 25-32, Sep. 2016.

[18] N. Miller, K. Clark, and R. Walling, "Winsinertia: Controlled inertial response from GE wind turbine generators," in 45th Annual Minnesota Power Systems Conference, Nov. 2009.

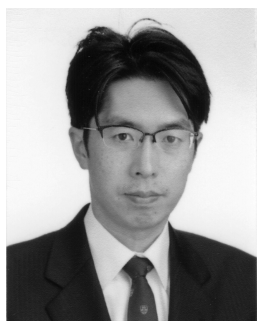

Koji Yamashita (M'04) received his B.S. and M.S. degrees from Waseda University, Tokyo, Japan, in 1993 and 1995, respectively. He is currently a Senior Research Scientist at the Central Research Institute of Electric Power Industry (CRIEPI) in Japan and has been with the Department of Power Systems since 1995 . He was a visiting researcher at Iowa State University during 2006-2007. He has been a co-convener of CIGRE C4-C6.35/CIRED JWG, "Modelling and Dynamic Performance of Inverter Based Generation in Power System Transmission and Distribution Studies" since 2013.

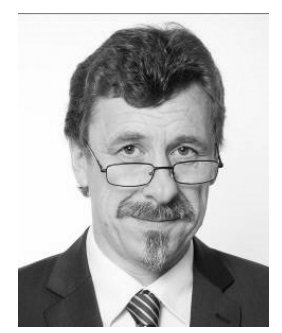

Herwig Renner works as associate professor at the Institute for Electrical Power Systems at Graz University of Technology. His main fields of interests are in the area of industrial power quality and in the power system control and stability. $\mathrm{He}$ is currently chairman of CIRED session 2, co-convener of CIGRE C4-C6.35/CIRED and member of several CIRED/CIGRE working groups.

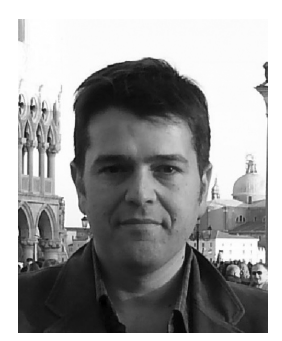

Sergio Martínez Villanueva received his Master degree in Industrial Engineering in Electrical major from Polytechnic University of Madrid. He works since 2005 for Red Elctrica de Espaa, the TSO of the Spanish electricity system, in the Power System Reliability Department. His key tasks are related to power system modelling and analysis.

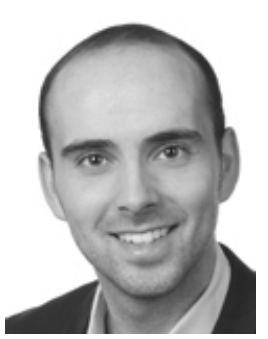

Gustav Lammert (S'13) received the B.Eng. degree in electrical engineering from the BadenWuerttemberg Cooperative State University, Mannheim, Germany, in 2010 and the M.Sc. degree in renewable energies and energy efficiency from the University of Kassel, Kassel, Germany, in 2013. He is now a Ph.D. student at the Dept. of Energy Management and Power System Operation, University of Kassel, Kassel, Germany. His fields of interest are modelling of PV systems, power system dynamics and stability.

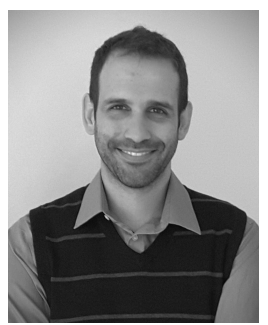

Petros Aristidou (S'10) obtained his Diploma in Electrical and Computer Engineering from the National Technical University of Athens, Greece, and his Ph.D. in Electrical Power Systems from the University of Liège, Belgium, in 2010 and 2015, respectively. He is currently a Lecturer (Assist. Prof.) in Smart Energy Systems at the University of Leeds, UK. His research interests include power system dynamics, control, and simulation. In particular, investigating the development and use of high performance computational tools in future power systems.

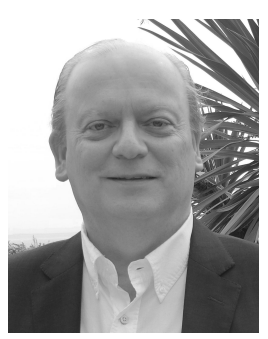

José Carvalho Martins received his Diploma (5 years) in Electrical Engineering and his Master degree in Electrical and Computer Engineering from the Technical University of Lisbon, in 1978 and 1987, respectively. He worked for 19 years as Junior Assistant, Assistant and Invited Assistant Professor, teaching Electrical Engineering university disciplines. He joined the EDP Group in 1992 and is currently a Specialist Engineer in the Asset and Network Planning Department of EDP Distribuio, the main DSO of the Portuguese electricity system. His fields of interest include planning of power distribution networks and cost-benefit optimization for long term investments.

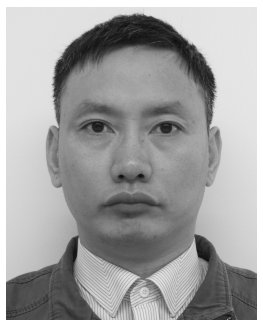

Lingzhi Zhu received the Ph.D degree from Tsinghua University, Beijing, China, in 2005. He joined China Electric Power Research Institute (CEPRI) in 2012 and now he is vise chief engineer of renewable energy research center of CEPRI. He has been a convener of IEC SC8A WG1, "Terms and definitions of grid integration of renewable energy generation" since 2016.

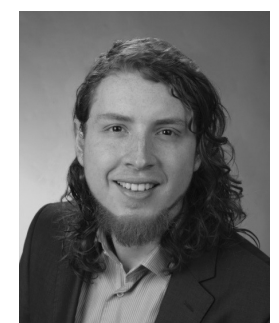

Luis David Pabón Ospina (M'16) received the B.Sc. and M.Sc. degrees in electrical engineering from Universidad Pontificia Bolivariana, Medellín, Colombia. He received his M.Eng. degree from the University of Kempten, Kempten, Germany. He is currently working toward the Ph.D. degree at the Dept. of Power System Control and Dynamics, Fraunhofer Institute for Energy Economics and Energy System Technology (IEE), Kassel, Germany.

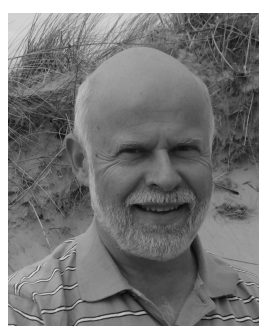

Thierry Van Cutsem (F'05) received the M.Sc. and $\mathrm{Ph} . \mathrm{D}$. degrees from the University of Liege, Belgium. He is currently a Research Director of the Fund for Scientific Research (FNRS) and an Adjunct Professor at the Dept. of Electrical Engineering and Computer Science at the same university. His research interests include power system dynamics, security, monitoring, control, and simulation. He served as Secretary, Vice-Chair, and Chair of the IEEE Power System Dynamic Performance Committee. 